\title{
Studies of Quadrupole Interactions by means of Angular Correlations*
}

\author{
P. Da R. Andrade, Alice Maciel, and J. D. Rogers \\ Instituto de Física, Universidade Federal do Rio Grande do Sul, Pôrto Alegre, Brasil
}

(Received 21 October 1966)

\begin{abstract}
Measurements of the nuclear quadrupole interaction have been made for the first $\frac{5}{2}^{+}$excited state of $\mathrm{Ta}^{181}$ in several compounds of $\mathrm{Hf}$, and for the first $\frac{5}{2}^{+}$excited state of $\mathrm{Cd}^{111}$ in In metal by time-differential and by integral-combined-field angular-correlation studies. Interaction frequencies derived from the two measurements are in reasonable agreement and demonstrate the static nature of the interactions involved. Quadrupole-interaction frequencies in $10^{6} \mathrm{rad} / \mathrm{sec}$ were determined as $\omega_{E}^{0}=6 \omega_{E}=(324 \pm 20),(\geq 600)$, $(\geq 600)$ for $\mathrm{Ta}^{181}$ in metallic $\mathrm{Hf}, \mathrm{HfOCl}_{2} \cdot 8 \mathrm{H}_{2} \mathrm{O}$, and $\mathrm{HfO}_{2}$, respectively, and $(39 \pm 10)$ for $\mathrm{Cd}^{111}$ in In metal by combined interaction measurements. A fundamental frequency of $\omega_{E}^{0}=(680 \pm 80)$ for $\mathrm{Ta}^{181}$ in $\mathrm{HfOCl}_{2}$. $8 \mathrm{H}_{2} \mathrm{O}$ was determined by a differential measurement. Limitations and possibilities of the two techniques are discussed.
\end{abstract}

\section{INTRODUCTION}

$\mathbf{T}$ HE experiments reported here were performed to provide an experimental comparison of techniques for the determination of nuclear quadrupole interactions in polycrystalline solids from angular-correlation studies.

Two major techniques have been used. The first was the measurement of the anisotropy of the integral angular correlation as a function of the external magnetic field strength (the "integral-combined-interaction technique"). This method essentially measures the ratio of the nuclear magnetic dipole and electricquadrupole interactions. The second was the study of the rotation of the angular-correlation pattern as a function of the time between the two radiations (the "differential technique"). This provides a direct determination of the quadrupole-interaction frequencies. A complete discussion of these techniques together with references to the theory can be found in the literature. ${ }^{1,2}$

Measurements with combined interactions have been made on the 133-482-keV cascade through the $\tau_{n}=15.4$ nsec intermediate state of $\mathrm{Ta}^{181}$ following the decay of $\mathrm{Hf}^{181}$ in the compound $\mathrm{HfOCl}_{2} \cdot 8 \mathrm{H}_{2} \mathrm{O}$ and in metallic hafnium, and on the 173-247-keV cascade through the $\tau_{N}=120 \mathrm{nsec}$ intermediate state of $\mathrm{Cd}^{111}$ following the decay of $\mathrm{In}^{111}$ in metallic cadmium. The differential correlation has been studied for the case of $\mathrm{HfOCl}_{2}$. $8 \mathrm{H}_{2} \mathrm{O}$ and for metallic $\mathrm{Hf}$. The study of the latter structure was done as a check, since previous data exist in the literature for this case as well as for $\mathrm{Cd}$ in metallic indium..$^{3,4}$ These studies provide a comparison of the two methods for a wide range of interaction strengths and give a good picture of the relative advantages of each.

\footnotetext{
* Work supported by the U. S. Army Research Office and Conselho Nacional de Pesquisas (Brasil).

1 Perturbed Angular Correlations, edited by E. Karlsson, E. Matthias, and K. Siegbahn (North-Holland Publishing Company, Amsterdam, 1964), Chap. I.

${ }^{2}$ Alpha-, Beta-, and Gamma-Ray Spectroscopy, edited by $\mathbf{K}$. Siegbahn (North-Holland Publishing Company, Amsterdam, 1965), Vol. II, Chap. XIX, Part II.

${ }^{3}$ R. W. Sommerfeldt, T. W. Cannon, L. W. Coleman, and L. Schecter, Phys. Rev. 138, B763 (1965).

${ }^{4}$ P. Lehmann and J. Miller, J. Phys. Radium 17, 526 (1956)
}

\section{THEORETICAL BACKGROUND}

The interaction of nuclear quadrupole moments with a static electric-field gradient in polycrystalline materials can be specified in terms of a fundamental frequency $\omega_{E}{ }^{0}$, and a parameter $\eta$ which describes the asymmetry of the electric field gradients. In addition, it is usually assumed that the field gradient acting on different nuclei may not be the same. This is taken into account in the theory by the introduction of a Gaussian distribution of frequencies specified by a parameter $^{5} \delta$. Some experimental evidence in support of this description has been reported.,

In the best cases it is possible to determine both $\omega_{E}{ }^{0}$ and $\eta$ quite accurately from differential measurements and to get an indication of the parameter $\delta$. The major difficulty in the experiment arises when the interaction frequency is so high that the finite resolving time $2 \tau_{0}$ of the coincidence system limits the resolution of the fine structure of the curve. This problem has been discussed ${ }^{7}$ and sets the restriction $\omega_{E}^{0} \tau \sim 1$ as a practical upper limit for the frequency when this technique is used. Typical theoretical curves for the cases of interest here have been published in several papers. $^{3,5,7}$

The integral-combined-interaction technique is considerably simpler technically, since it does not require a coincidence apparatus with very fast time resolution. The results depend essentially on the parameters $x=\omega_{E} \tau_{N}$, where $\tau_{N}$ is the mean life of the nuclear state, and $y=\omega_{H} / \omega_{E}$, the ratio of magnetic-dipole and electricquadrupole interaction strengths. ${ }^{8}$ The most important limitation is that for high interaction strengths $\omega_{H}$ must be comparable to $\omega_{E}$ before significant effects are observed, which often may require magnetic fields larger than those easily obtainable in laboratory conditions.

Two particular geometrical arrangements are inter-

${ }^{5}$ E. Matthias, W. Schneider, and R. M. Steffen, Phys. Letters 4, 41 (1963).

${ }^{6}$ L. Mayer, E. Bodenstedt, and C. Günther, Z. Physik 177, 28 (1964).

7 M. Salomon, L. Boström, T. Lindqvist, M. Perez, and M. Zwanziger, Arkiv Fysik 27, 97 (1964).

${ }^{8}$ K. Alder, E. Matthias, W. Schneider, and R. M. Steffen, Phys. Rev. 129, 1199 (1963).

196 
FIG. 1. Theoretical curves for the anisotropy as a function of magnetic field $H$ for the $\frac{7}{2} \rightarrow \frac{5}{2} \rightarrow \frac{1}{2}$ cascade in the decay of $\operatorname{In}^{111}$ under the influence of combined magnetic and electric interactions for various values of $x=\omega_{E} \tau_{N}$.

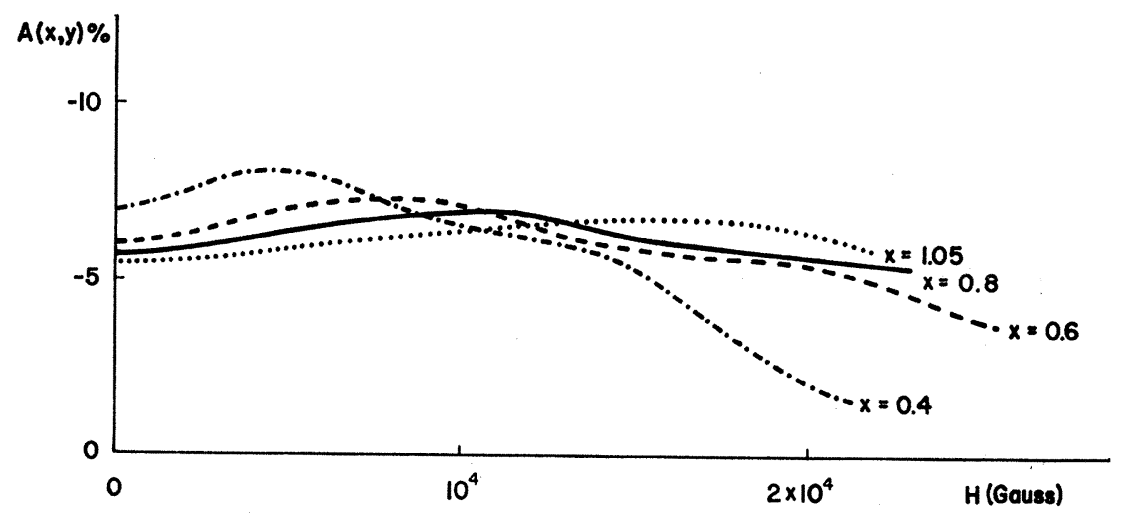

esting for combined-interaction studies. In the first, the magnetic field is directed parallel to the direction of one of the $\gamma$ rays (the so-called decoupling experiment), whereas in the second (the arrangement used in these experiments), the field is directed perpendicular to the plane of the correlation measurement. In this latter arrangement, the anisotropy of the angular correlation is given by the expression ${ }^{1}$

$$
\begin{aligned}
& A_{\perp}(x, y)= {\left[60 A_{22} a_{22}{ }^{(2)}-30 \sqrt{3}\left(A_{24}+A_{42}\right) a_{24}{ }^{(2)}\right.} \\
&\left.+45 A_{44} a_{44}{ }^{(2)}\right]\left[16(2 I+1)+10 A_{22}\left(a_{22}{ }^{(0)}-3 a_{22}{ }^{(2)}\right)\right. \\
&+\frac{9}{2} A_{44}\left(\frac{9}{4} a_{44}{ }^{(0)}-5 a_{44}{ }^{(2)}+(35 / 4) a_{44}{ }^{(4)}\right) \\
&\left.-\frac{3}{2}\left(A_{24}+A_{42}\right)\left((3 \sqrt{ } 5) a_{24}{ }^{(0)}-10 \sqrt{3} a_{24}{ }^{(2)}\right)\right]^{-1},
\end{aligned}
$$

where the quantities $A_{k_{1} k_{2}}$ are the angular-correlation coefficients and depend on the parameters of the

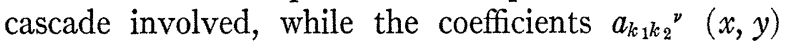
are the real parts of the perturbation coefficients and depend on the spin of the intermediate state and on the strength of the static perturbing fields as expressed by $x$ and $y$.
We have used this formula to calculate some theoretical curves for the anisotropy of the studied correlations in $\mathrm{Cd}^{111}$ and $\mathrm{Hf}^{181}$. The numerical values of $a_{k_{1} k_{2}}{ }^{\nu}$ $(x, y)$ were taken from Steffen, Matthias, and Schneider ${ }^{9}$ and are for the case $\eta=0$. (As has been shown, $, 5,10$ the time-integrated curves are virtually independent of $\delta$ and $\eta$.) The results of this calculation are shown in Figs. 1 and 2 and illustrate the qualitative features mentioned above.

\section{APPARATUS}

The differential measurements were performed with differential equipment consisting ${ }^{11}$ basically of a transistorized time-to-pulse-height converter and a 256channel Nuclear Data Analyzer. The detectors were two 6810-A RCA photomultipliers with a 2 -in. $\times 1.5$-in. $\phi$ lead-loaded plastic scintillator for the low-energy $\gamma$ and a 2 -in. $\times 1.5$-in. $\phi \mathrm{NaI}(\mathrm{Tl})$ crystal for the highenergy $\gamma$.

The total time range in the differential measurements was 100 nsec and the full width at half-maximum
Fig. 2. Theoretical curves for the anisotropy as a function of magnetic field $H$ for the $\frac{1}{2} \rightarrow \frac{5}{2} \rightarrow \frac{7}{2}$ cascade in the decay of $\mathrm{Hf}^{181}$ under the influence of combined magnetic and electric interactions for various values of $x=\omega_{E} \tau_{N}$.

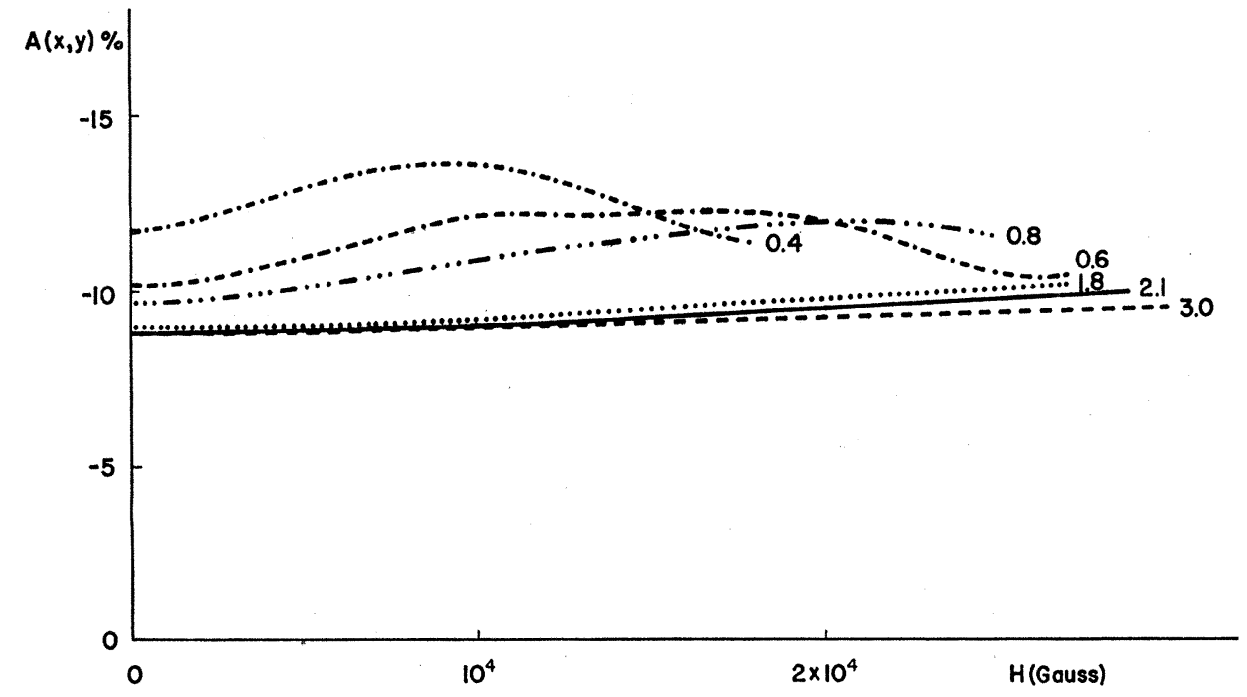

9 R. M. Steffen, E. Matthias, and W. Schneider, AECTID Report No. 17089 (unpublished).

${ }^{10} \mathrm{E}$. Matthias, B. Olsen, and W. Schneider, Arkiv Fysik 24, 245 (1963).

${ }^{11}$ D. E. Brandão, H. J. Körner, A. Maciel, C. S. Müller, and F. C. Zawislak, Nucl. Phys. 56, 65 (1964). 


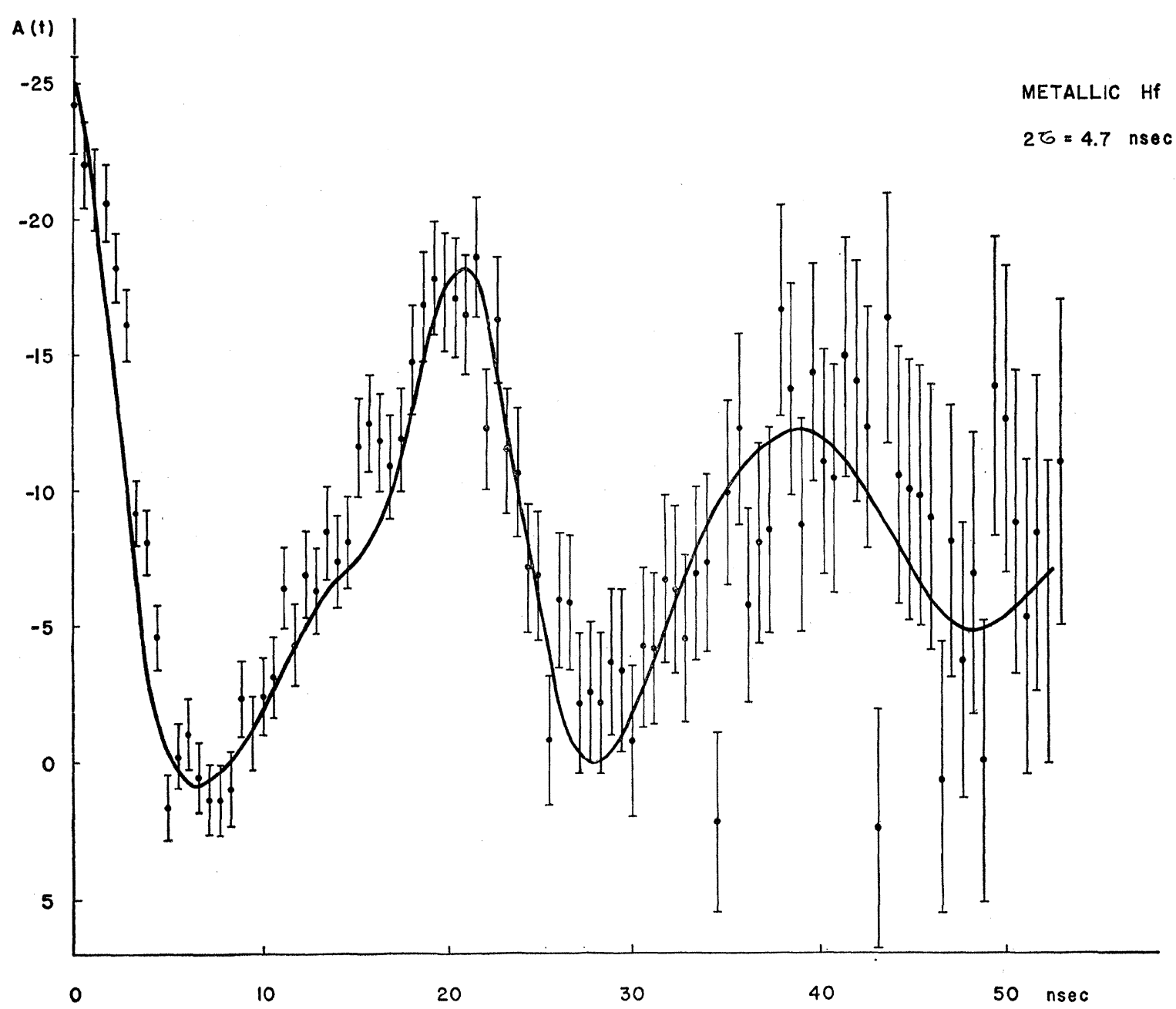

Fig. 3. Experimental anisotropy of the 133-482 $\gamma-\gamma$ cascade with metallic Hf obtained with $2 \tau_{0}=4.7 \mathrm{nsec}$. The solid line is the theoretical curve for $\omega_{E}{ }^{0}=299 \times 10^{6} \mathrm{rad} / \mathrm{sec}, \eta=0.30, \delta=10 \%$, and was taken from Ref. 3 .

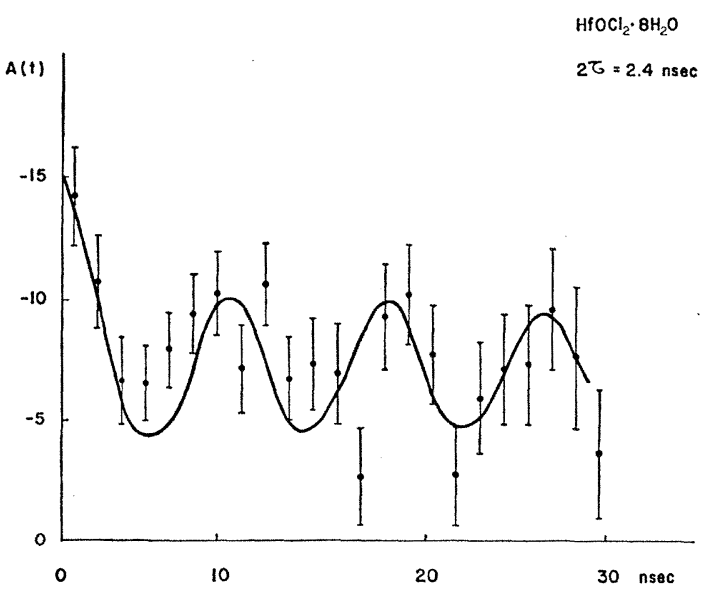

Fig. 4. Experimental anisotropy of the 133-482 $\gamma-\gamma$ cascade with $\mathrm{HfOCl}_{2} \cdot 8 \mathrm{H}_{2} \mathrm{O}$ obtained with $2 \tau_{0}=2.4$ nsec. The solid line is the theoretical curve for $\omega_{E}{ }^{0}=700 \times 10^{6} \mathrm{rad} / \mathrm{sec}, \eta=0, \delta=4 \%$, and was taken from Ref. 7 . of the prompt peak (taken with $\mathrm{Na}^{22}$ with the energy settings for the $\mathrm{Ta}^{181}$ cascade) was $4.7 \mathrm{nsec}$ for the metallic $\mathrm{Hf}$ experiment and $2.4 \mathrm{nsec}$ for the $\mathrm{HfOCl}_{2}$. $8 \mathrm{H}_{2} \mathrm{O}$ experiment.

The integral-combined-interaction measurements were performed with integral equipment described elsewhere. ${ }^{12}$ The resolution of the fast coincidence system was 120 nsec.

In all measurements, the room temperature, the line voltage, and the current of the electromagnet were controlled so as to obtain an over-all stability better than $0.5 \%$.

The $\mathrm{Hf}^{181}$ radioactive source was obtained from the reactor of the Instituto de Energia Atômica in São Paulo, irradiating metallic Hf, and the $\operatorname{In}^{111}$ source was obtained from the Buenos Aires cyclotron through the reaction $\mathrm{Cd}^{110}(d, n) \mathrm{In}^{111}$ in natural cadmium.

${ }^{12}$ P. da R. Andrade, A. Maciel, C. S. Müller, J. Wirth, and F. C. Zawislak, Nucl. Phys. 66, 545 (1964). 
Fig. 5. Comparison of the experimental integral anisotropy for metallic $\mathrm{Hf}$ and $\mathrm{HfOCl}_{2} \cdot 8 \mathrm{H}_{2} \mathrm{O}$ as a function of magnetic field with theoretical curves for a static combined magnetic dipole and electric quadrupole interaction.

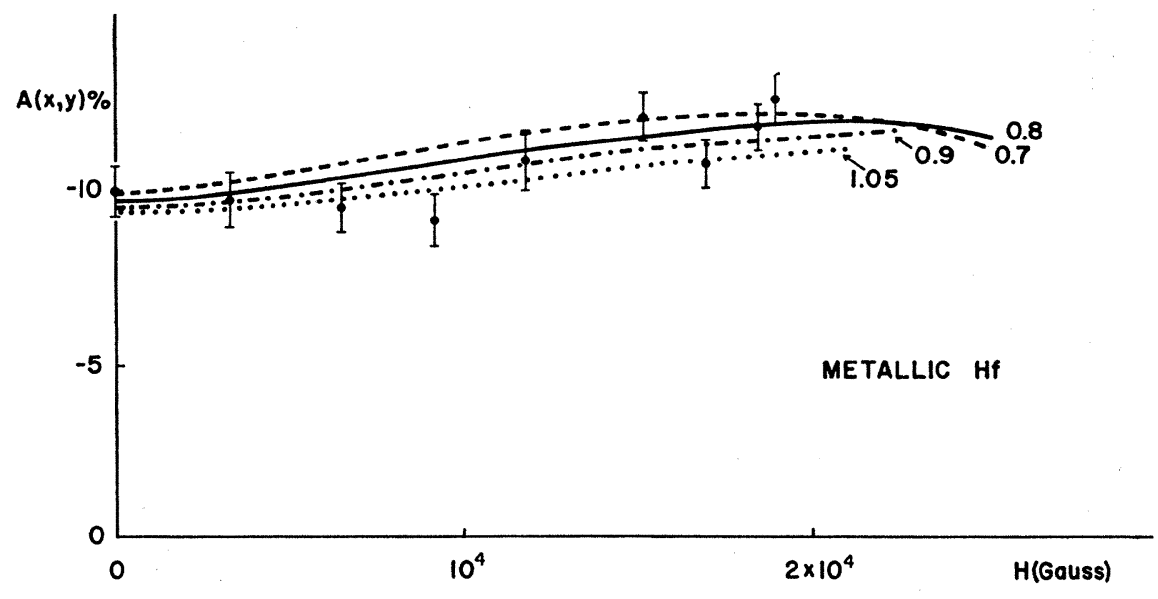

(a)

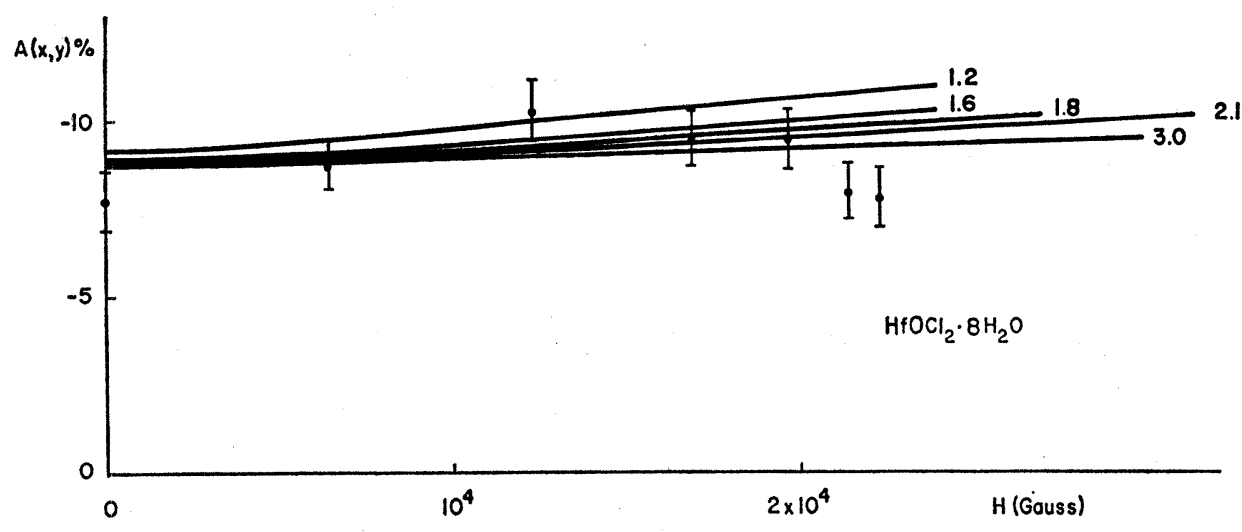

(b)

\section{RESULTS AND CONCLUSIONS}

The results of the differential measurements made on metallic $\mathrm{Hf}$ and $\mathrm{HfOCl}_{2} \cdot 8 \mathrm{H}_{2} \mathrm{O}$ are shown in Figs. 3 and 4. The results for metallic Hf, which was measured as a general test of the equipment, are compared to the curve derived by Sommerfeldt $e t a l{ }^{3}$ as a best fit to their measurements. The agreement with this curve, which is specified by $\delta=10 \%, \quad \eta=0.30 \pm 0.03, \quad \omega_{E}{ }^{0}=$ $(299 \pm 8) \times 10^{6} \mathrm{rad} / \mathrm{sec}$, can be seen to be excellent. The appearance of a shoulder on the initial peak of the correlation pattern as a result of the asymmetry of the electric-field gradient is quite obvious. This is a typical case in which the full power of the differential technique can be seen. On the other hand, the results for HfOCl $\cdot 8 \mathrm{H}_{2} \mathrm{O}$ are at the limit of the frequency range which can be measured with this equipment. The strong effects of the finite time resolution $\left(2 \tau_{0}=2.4 \mathrm{nsec}\right)$ in smearing out the details of the curve can be observed. The results are fitted to a curve reported by Salomon et al. ${ }^{7}$ with $\eta=0, \delta=4 \%, \omega_{E}{ }^{0}=(680 \pm 80) \times 10^{6} \mathrm{rad} / \mathrm{sec}$, but the values of $\eta$ and $\delta$ derived for this case are only qualitative.

In Figs. 5 and 6 the results of the combined-inter- action experiments for metallic $\mathrm{Hf}, \mathrm{HfOCl}_{2} \cdot 8 \mathrm{H}_{2} \mathrm{O}$, and metallic In are shown. Values of the parameter $x$ have been derived for each case by a one-parameter leastsquares fitting ${ }^{13}$ of the experimental points to the theoretical curves shown in Figs. 1 and 2. The error has been chosen so as to include all fits for which the calculated $\chi^{2}$ was less than $(n-1)$, where $n$ is the number of measured points (see, e.g., Ref. 13 for a detailed discussion). The values of $x=\omega_{E} \tau_{N}$ and of $\omega_{E}^{0}=6 \omega_{E}$ thus obtained are summarized in columns 2 and 3 of Table

TABLE I. Calculated values of the fundamental frequencies and derived values of electric-field gradients for the chemical compounds of $\mathrm{Hf}$ and metallic In. In evaluating the electric-field gradients, the values of $Q=(2.5 \pm 0.10) 10^{-24} \mathrm{~cm}^{2}$ and of $Q \approx 10^{-24}$ $\mathrm{cm}^{2}$ were used for $\mathrm{Ta}^{181}$ and $\mathrm{Cd}^{111}$, respectively (Refs. 1 and 14 ).

\begin{tabular}{|c|c|c|c|}
\hline Sources & $\begin{array}{c}x=\omega_{E} \tau_{N} \\
(\mathrm{rad})\end{array}$ & $\begin{array}{c}\omega_{E}^{0}=6 \omega_{E} \\
\left(10^{6}\right. \\
(\mathrm{rad} / \mathrm{sec})\end{array}$ & $\begin{array}{l}\left|V_{z z}\right| \\
\left(\mathrm{V} / \mathrm{cm}^{2}\right)\end{array}$ \\
\hline $\begin{array}{l}\text { Hf metal } \\
\mathrm{HfOCl}{ }_{2} \cdot 8 \mathrm{H}_{2} \mathrm{O} \\
\text { In metal }\end{array}$ & $\begin{array}{l}0.85 \pm 0.05 \\
\geq 1.6 \\
0.80 \pm 0.20\end{array}$ & $\begin{array}{c}324 \pm 20 \\
\geq 610 \\
39 \pm 10\end{array}$ & $\begin{aligned}(5.6 \pm 0.3) \times 10^{17} & \geq 11 \times 10^{17} \\
& \approx 1.7 \times 10^{17}\end{aligned}$ \\
\hline
\end{tabular}

${ }^{13}$ A. J. Ferguson, Angular-Correlation Methods in Gamma-Ray Spectroscopy (North-Holland Publishing Company, Amsterdam, 1965), Chap. 4. 


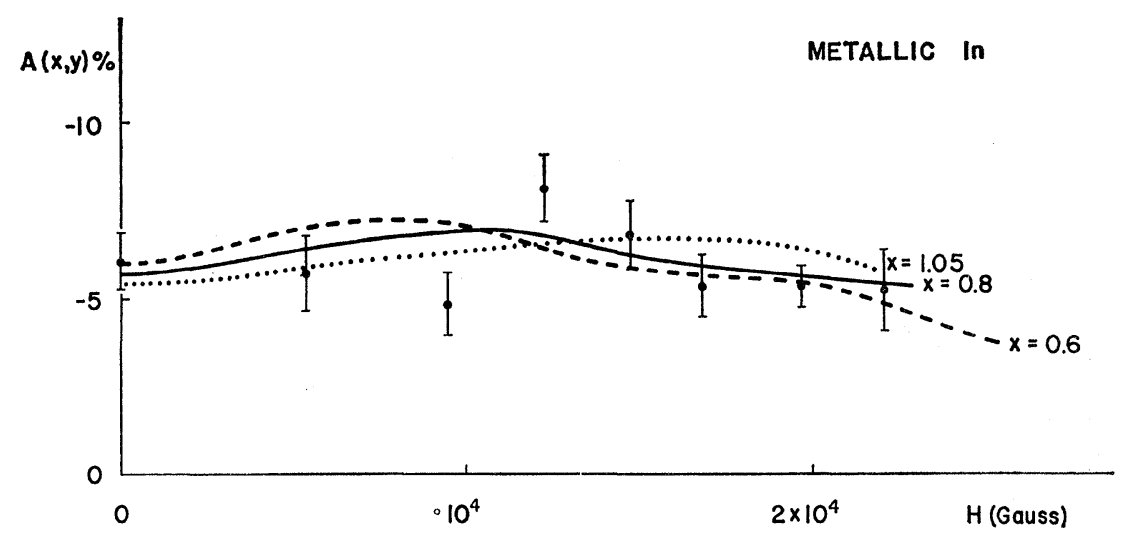

FIG. 6. Comparison of the experimental integral anisotropy for metallic In as a function of magnetic field with theoretical curves for a static combined magnetic dipole and electric quadrupole interaction.

I. ${ }^{14}$ In column 4, the values of the axial component of the electric-field gradient deduced from the values of $\omega_{E}{ }^{0}$ are given using the known values of the quadrupole moments.

In addition to the above results, we have performed preliminary combined-interaction experiments on $\mathrm{HfO}_{2}$ which show a behavior similar to that of $\mathrm{HfOCl}_{2} \cdot 8 \mathrm{H}_{2} \mathrm{O}$. The resulting high interaction frequency $\left(\omega_{E}{ }^{0} \geq 610 \times 10^{6}\right.$ $\mathrm{rad} / \mathrm{sec}$ ) is in good agreement with that found from differential experiments by Marest et $a .^{15}$ and by Salomon $^{16}$ and is consistent with the low decoupling effect for this compound observed by Matthias et $a l .^{17}$

The results for metallic Hf shown in Fig. 5 may be compared with the values $x \approx 0.9$ and 0.6 obtained by Matthias et $a l .^{18}$ for two different sources of metallic hafnium with fields ranging up to $50 \mathrm{kG}$. It is clear that oxidation of the hafnium can not account for this discrepancy since the interaction in $\mathrm{HfO}_{2}$ is very large. Our result of $x \approx 0.8$ is in good agreement with results of the differential measurements reported above.

The results shown for all three of these compounds follow quite well the static theory, indicating that possible time-dependent effects such as those observed ${ }^{17,19}$ for $\left(\mathrm{NH}_{4}\right)_{3} \mathrm{HfF}_{7}$ are not present to a significant extent in these sources. For $\mathrm{Hf}$ and $\mathrm{Cd}$ metals, rather accurate values of the fundamental electrostatic quadrupole frequency could be obtained from the combined-interaction experiments. On the other hand, for $\mathrm{HfOCl}_{2} \cdot 8 \mathrm{H}_{2} \mathrm{O}$ the very high interaction strength causes the curve to approach very close to the hard-core limit and only a lower bound can be given for the quadrupole frequency. In all cases, the results are in good agreement with the various measurements by the differential technique for the values of $\omega_{E}{ }^{0}$. It is interesting to note that although the $x$ values found for In and Hf metals are the same, the factor of 10 in the mean-life results

${ }^{14}$ P. H. Stelson and F. K. McGowan, Phys. Rev. 105, 1346 (1957).

${ }^{15}$ G. Marest, I. Berkes, G. Bougnot, and R. Beraud, Compt. Rend. 262, B367 (1966).

${ }^{16} \mathrm{M}$. Salomon (private communication).

17 E. Matthias, E. Karlsson, A. G. Svensson, K. Johansson, and P. da R. Andrade, in Ref. 1, p. 275.

${ }_{18}$ E. Matthias, E. Karlsson, A. G. Svensson, K. Johansson, and S. Gustafsson, Arkiv Fysik 30, 19 (1965).

${ }_{19}$ M. Deutsch, A. Hrynkiewicz, and R. Stiening, MIT Laboratory of Nuclear Science Progress Report, 1959 (unpublished). in a much lower frequency for metallic $\mathrm{Cd}$. This relatively low frequency leads us to suggest that this source would be an excellent candidate for a parallel-decoupling experiment.

The upper limit of the combined-interaction method is determined by the fact that for large values of $x$ significant structure in the curve of $A(H)$ is only obtained for values of $\omega_{E} \approx \omega_{H}$. This sets a practical upper limit of the order of $500 \times 10^{6} \mathrm{rad} / \mathrm{sec}$ for $\omega_{E}^{0}$ in our experimental conditions. This limit is of the order of magnitude of that imposed in the differential measurements as a result of the finite time resolution of the coincidence system. Since, in principle, more information is obtainable from the differential experiments, this would seem to argue in their favor in this range of frequencies. There are, however, several cases in which the integral technique may be preferable. Some cases might occur, for example, when the mean life of the intermediate state is short or where electronic difficulties prevent the obtaining of a short resolving time because of the low energy of one of the radiations, for example, or of the type of detector involved.

The comparison of the two results may also be of considerable importance in separating time-dependent effects from effects due to frequency distributions or asymmetries of the field gradients [see, for example, the results ${ }^{17,19}$ for $\left.\left(\mathrm{NH}_{4}\right)_{3} \mathrm{HfF}_{7}\right]$. We conclude from these measurements that the integral-combined-interaction technique can be a powerful and useful adjunct to the study of crystalline properties by the usual angularcorrelation techniques. Especially when used together with the differential technique, it can provide valuable evidence as to the magnitude and nature of the fields acting on the nucleus due to its atomic and crystalline environment.

\section{ACKNOWLEDGMENTS}

The authors thank E. R. Fraga for the preparation of the sources and one of them (P.da R. A.) expresses his gratitude to F. C. Zawislak for his orientation in the operation of the differential angular-correlation experiments. One of the authors (J.D.R.) is indebted to the Pan American Union for support during a part of this work. The encouragement of Gerhard Jacob during the work is gratefully acknowledged. 